\title{
Host Modulation- Promising Direction In Periodontal Therapy
}

\author{
Dr.Priyadarshni Rangarajan ${ }^{1}$, Dr.Hariharavel Vp ${ }^{2}$, Dr.Ieshwaryah Kumanan ${ }^{3}$ \\ ${ }^{\prime}$ (Senior Lecturer, Department of Periodontics, SRM Kattankulathur Dental College, Chennai) \\ ${ }^{2}$ (Senior Lecturer, Department of Pedodontics and Preventive Dentistry, SRM Kattankulathur Dental College, \\ Chennai) \\ ${ }^{3}$ (Lecturer, Department of Pedodontics and Preventive Dentistry, SRM Kattankulathur Dental College, \\ Chennai)
}

\begin{abstract}
The primary etiology of the periodontal disease is chronic inflammation due to bacterial infection. It is the host's reaction to the presence of bacteria that mediates tissue destruction . Since the destruction of periodontium is believed to be due to the host response, it is intellectual and logical to consider therapeutic approaches that modulate the host response in addition to antibacterial approaches in periodontal therapy. Improved knowledge of bacterium -host interactions and of the process leading to tissue destruction will help to identify targets for host modulation to reduce periodontitis in selected situations. The objective of this article is to review and update the fundamental scientific concepts of host modulation and to furnish an approach to the main types of therapy used, based on the scientific literature.
\end{abstract}

KEYWORDS : Periodontitis, Anti-Inflammatory, Host Response, Pathogenesis, Adjunctive Therapy.

\section{INTRODUCTION}

In recent years, there is a paradigm shift in our understanding of the etiopathogenesis of periodontal disease. Periodontal disease is considered as an inflammatory disease initiated by tooth associated biofilm. However, the progression of the disease and destruction of periodontal tissues is due to series of complex interactions between micro biota and host immune responses[1]. It is becoming clear day to day that inflammation is the major determinant of susceptibility for periodontitis. But our understanding of relationship of etiologic agents of periodontitis to the pathogenesis is not as clear as it was once thought. Today the clinical management of periodontal disease is based on 3 steps:

- Reduction of the bacterial burden.

- Modification of environmental and behavioral risk factors.

- Modulation of host responses.

With the current understanding of the host response and periodontal disease pathogenesis, it is intuitive that pharmaceutical inhibition of host response pathway may be an adjunctive or alternative strategy for treating periodontal disease.

\section{HOST MODULATION}

Host modulation means modulating destructive or damaging aspects of inflammatory response that develops in face of the challenge presented by the subgingival bacterial plaque. Host modulation is not a new concept. However, in medicine the host modulation stratergies are successfully used in the treatment of disorders such as rheumatoid arthritis and osteoporosis. In periodontitis a variety of pharmacological agents have been studied for possible role as host modulators in prevention and management of periodontal disease. These include non steroidal anti-inflammatory drugs, bone sparing agents, anticytokine drugs and anti matrix metallo proteinases.

\subsection{Non Steroidal Anti-Inflammatory Drugs:}

Arachidonic acid is liberated from plasma membrane phospholipids via the enzyme phospholipase- 2 . Free arachidonic acid can be metabolized to cyclooxygenase or lipoxygenase pathways. Cycloxygenase-2 is inducible and thought to be involved in inflammation, cellular differentiation and mitogenesis. Cycloxygenase 1 and cycloxygenase-2 produce prostaglandin[2], prostacyclin and thromboxin whereas lipoxygenases produces leukotrienes and other hydroxyl eicosatetraenoic acids[3]. And these enzymes have been implicated in wide range of events in inflammatory diseases. Anti-inflammatory drugs block the cycloxygenase pathways and inhibit inflammation. 
Utilizing canine experiment periodontitis model Nyman[4] and associates investigated the modulation of arachidonic acid metabolites with systemic indomethacin and demonstrated that systemic doses of non steroidal anti-inflammatory drugs suppresses alveolar bone resorption and gingival inflammation. Similar findings have been reported in ligature-induced periodontitis in squirrel, monkeys with systemic administration of indomethacin. Non steroidal anti inflammatory drug flurbiprofen [5]successfully blocks the progression of recurring periodontal disease in animal models. Propionic acid derivative ibuprofen was effective in blocking alveolar bone loss in naturally occurring periodontitis among beagle dogs[6]. Topical application of piroxicam in gel and liquid forms significantly reduced gingivitis in a preclinical trial [7]. 1\% ketoprofen cream has also shown similar results in rhesus monkey model [8]. Concurrently there is substantial data from human cross sectional and cohort studies indicating periodontal disease inhibition with non steroidal anti-inflammatory drugs. Waite et al[9] have noticed lower gingival index and shallower pocket depth in patients who are on non steroidal anti-inflammatory drugs for arthritis and ankylosing spondylitis compared to individuals not taking non steroidal anti-inflammatory drugs. In a cohort study 75 patients who are on aspirin and indomethacin [10] for arthritis had significantly fewer sites with proximal bone loss as compared to healthy controls. Jeffcoat et al [11] have reported less alveolar bone loss in patients who are on systemic flurbiprofen (15mg bid) in compared to placebo treatment. Systemic Naproxen [12] along with mechanical debridement in rapidly progressing periodontitis has shown significant reduction in inflammatory periodontal condition. In another study, mechanical scaling and acetyl salicylic acid resulted in synergistic reduction in gingival inflammation, probing pocket depth and clinical attachment loss. The non steroidal anti-inflammatory drugs suffer from serious disadvantages that essentially preclude their use as an adjunctive treatment for periodontal disease. Daily administration of these drugs for extended period of time is associated with significant unwanted effects including gastrointestinal problems, hemorrhage, renal and hepatic impairments. Once the drugs are withdrawn there is going to be a rebounding effect resulting in severe tissue damage.

\subsection{Cytokine Inhibitors:}

Cytokines means, "cell protein". They transmit information from cell to cell via autocrine and paracrine mechanism. The cytokines can be proinflammatory or anti-inflammatory. IL-1 $\beta$ and TNF- $\alpha$ are known for their role in periodontal inflammation and tissue destruction. Studies have shown that the GCF levels of these cytokines increases in periodontal disease [13]. Following specific binding to the receptors on the cell surfaces, IL-1 $\beta$ and TNF- $\alpha$ trigger intercellular signaling events and catabolic cell behavior. To counterbalance the catabolic activity of cytokines and to maintain homeostasis, both IL- $1 \beta$ and TNF- $\alpha$ have endogenous inhibitors. In addition, the IL-1b level increases rapidly in experimental gingivitis $[14,15]$, and IL$1 \mathrm{~b}$ is produced by a variety of cells in the periodontal area, including monocytes, gingival fibroblasts[16] , and PMNs [17] . IL-1b regulates the fibroblast phenotype [18], including increases in collagenase production[19,20] and decreases in collagen production from gingival fibroblasts[21]. The IL-1 receptor antagonist which has a structure related to IL-1, compete with IL-1 to bind with its receptors without triggering signal transduction. These endogenous inhibitors also occur in soluble from as competitive antagonist of IL-1 and TNF $\alpha$. This receptor antagonist has been used successfully in many inflammatory conditions to modulate the host response. In non human primates, ligature induced periodontitis progression has been partially inhibited. However, the use of cytokines antagonist to treat human disease has to be evaluated further, because of many unresolved issues [22]. Importance among them are systemic implication associated with altering cytokines levels on tissue homeostasis and the method of administrating and maintaining cytokine levels for a long time. Anti inflammatory cytokines IL- 4, IL-10, IL-1 [23] and transforming growth factor $\beta$ (TGF- $\beta$ ) have been implicated in suppressing destructive inflammatory response. IL-4 and IL-10 target macrophages and inhibit the release of IL-1, TNF- $\alpha$ and reactive oxygen species. IL-4 is also known to induce apoptosis-programmed cell death that reduces the number of infiltrated macrophages. IL-4 is also shown to up regulate production of IL-1 receptor antagonist.

Exogenous IL-4 has been administered to modulate the inflammatory response in experimental arthritis and it has been observed that IL-4 reduces the inflammatory response [24]. Recombinant IL-11 is reported to inhibit TNF $\alpha$, IL-1 and nitric oxide production and reduce disease progression in ligature induced periodontal canine model [25]. Granulocyte colony stimulating factor (GCSF) has been reported in a 15 year long study in improving periodontal condition in cases of cyclic neutropenia [26].

\subsection{Bone sparing agents:}

The therapeutic strategies used to prevent and manage osteoporosis, osteopenia may also inhibit periodontal bone loss. Bisphosphonates are non biodegradable analogue of pyrophosphate, that have high affinity for calcium phosphate crystals and that inhibit osteoclast activity [27] .These compounds also appear to inhibit MMP activity through a mechanism that involves chelation of cations. They are used successfully in 
the clinical management of osteoporosis. One of these drugs Alendronate has been evaluated in ligature induced periodontitis model and assessed for changes in bone density [28]. Alendronate inhibited the loss of bone density in these models. However, minimal effects were demonstrated on clinical parameter. Studies using topical administration of bisphosphonates have reported reduction in bone and root resorption associated with orthodontic tooth movement [29,30]. However, further randomized control longitudinal studies are required to evaluate the effectiveness as well as side effects of these drugs in the treatment of the periodontal disease.

\subsection{Lipoxin, resolvins and protectins:}

Various inflammatory mediators like arachidonic acid metabolites and cytokines are released as host defense during periodontal infection to counteract the bacteria. But the recent concept of host bacterial interactions suggest that more than the destruction by bacterial virulence, it is the failure to resolve and heal is a cause of local tissue destruction. Resolution is a biological pathway of restoring homeostasis. Proresolving lipid mediators [31] are a family of novel endogenous chemical mediators, including lipoxins, resolvins and protectins . They are actively biosynthesized in the resolution phase of inflammation and are potent agonists that control the duration and magnitude of inflammation. Lipoxins (lipoxygenase interaction products) were the first proresolving mediators to be recognized. There are three major pathways for lipoxins generation. The first pathway involves the insertion of molecular oxygen to carbon 15 of arachidonic acid and implies the involvement of 15 lipoxygenase (15- LO). The products of 15- LO in epithelial cells and monocytes serve as substrates for neutrophil 5-LO and lead to the formation of trihydroxytetraenes, lipoxins A4. The second pathway involves interactions that occur within the vasculature between 5-lipoxygenase, present in myeloid cells and 12- lipoxygenase present in platelets.

The 5-LO leukotriene A4 is rapidly taken up by platelets and converted via 12-LO-dependent mechanism into lipoxins. The third major pathway for lipoxins generation involves aspirin and the actions of cyclo-oxygenase (COX-2) and 5-LO. Endothelial and epithelial cells express COX-2 in response to diverse stimuli such as cytokines, hypoxia and bacterial infections [32]. Aspirin acetylates COX-2 and switches its catalytic activity for the conversion of arachidonic acid (C20:4) to 15R -hydroxyeicosatetraenoic acid (15RHETE) in lieu of prostanoid biosynthesis. 15R-HETE is released from endothelial and epithelial cells and transformed by leukocyte 5-LO, via transcellular routes, to 15-epimer lipoxins or aspirin triggered lipoxins (ATLs), which is more stable and longer acting than the endogenously produced lipoxins. Kim et al[33] in a human study assessed the short term effects (7 days) of the systemic administration of two different concentrations of Aspirin ( 81 and $325 \mathrm{mg} / \mathrm{day}$, by mouth) on clinical periodontal parameters (probing depth, plaque index and bleeding on probing) and GCF levels of 15-epi-LXA4, LXA4, LTB4, PGE2, IL-6 and IL-1 $\beta$ in a sample of naturally occurring gingivitis patients using ELISA. They reported that the concentration of 15-epiLXA4 was 500- fold higher than the concentration of naturally occurring LXA4. They observed a negative trend between 15-epi-LXA4 and PGE2 (though it was not statistically significant) and concluded that subjects who possessed higher sensitivity to the aspirin mediator anti-inflammatory effects produced more 15-epi-LXA4. In an animal study Pouliot $M$ et al [34] investigated the impact of LXA4 and ATL in TNF- $\alpha$ initiated PMN responses in vitro and in vivo using LX analogs that are metabolically more stable. They reported that at nanomolecular levels, ATL and LXA4 analogs blocked TNF- $\alpha$ stimulated IL-1 $\beta$ release by isolate human PMN in vitro. In addition when these analogs were added to murine air pouches dramatically inhibited TNF- $\alpha$ stimulated leukocyte trafficking in vivo and concomitantly stimulated IL-4 in pouch exudates. New classes of proresolving lipid mediators have been identified in addition to endogenously derived lipoxins and aspirin triggered lipoxins. Such lipid molecules include resolvins and protectins which are derived from omega 3 poly unsaturated fatty acids, eicosapentanoic acid (EPA) and docosahexanoic acid (DHA).EPA is metabolized to resolvin E series and DHA gives rise to resolvin D series and protectins.

\subsection{MMP inhibitors:}

MMPs are family of gene dependent enzymes play a key role in tissue destruction of periodontal disease. MMPs are secreted by majority of cell types in periodontium including macrophages, neutrophils, fibroblasts, keratinocytes, endothelial cells \& osteoclasts. These enzymes are capable of degrading a variety of extracellular matrix molecules including collagens [35]. In healthy tissues MMPs are produced primarily by fibroblast $\&$ are concern with the maintenance of periodontal connective tissue. During inflammation there will be up regulation of MMP gene transcription resulting in increased production of MMPs. Regulation of MMP activity involves specific, endogenous tissue inhibitors of MMPs (TIMP) \& $\alpha$ macro globulins. They form complexes with active MMPs \& their latent precursors, there by inactivating their action. In periodontal disease MMP-8 \& MMP-9 is reported to rise in tissue and GCF[36]. Tetracyclines have the ability to down regulate the expression of MMPs. Today doxycyclin, a tetracycline is the only systemic host response modulator specifically indicated as an adjunctive treatment for periodontitis, and it is approved by US food and drug 
administrations for this purpose. Clinical studies have shown that 20mg dose of doxycycline hydrate taken twice daily for period of 3-9 months as an adjunct to root surface instrumentation has shown clinical significant improvement in probing depths \& attachment levels compared to patients treated by root surface instrumentation alone. These data demonstrate a very clear benefit of sub antimicrobial dose doxycycline when combined with appropriate and effective conventional therapy. Golub et al[37]showed that a 2 month regimen of SDD significantly decreased both the level of bone type collagen breakdown products and MMP- 8 and MMP-13 enzyme levels in chronic periodontitis patients. There is evidence that adjunctive $20 \mathrm{mg}$ subantimicrobial dose doxycycline, taken twice daily over 9 months, is beneficial in the treatment of chronic periodontitis.

\section{CONCLUSION}

It is now understood that, though the primary etiologic factor for periodontal disease is bacteria, the excessive host inflammatory response and / or inadequate resolution of inflammation is critical to the pathogenesis of periodontitis. A class shift is now apparent in management of periodontal disease with the introduction of proresolving compounds such as lipoxins, and their more stable bioactive form ATLs. More recently resolvins and protectins have shown that stimulating resolution pathways go a long way in restoring tissue homeostasis and periodontal healing. To this day only MMP inhibitor, Sub Antimicrobial Low Dose Doxycycline (SDD)has been permitted by FDA in treatment of periodontitis as an adjunctive to conventional therapy. There are many molecules which are being studied and may emerge in future as effective agents of host modulation and treatment of periodontal disease and this direction of research promise a paradigm shift in drug invention and therapeutics for periodontal disease.

\section{REFERENCES}

[1] Page RC, Kornman KS. The pathogenesis of human periodontitis: An introduction. Periodontol 2000 1997; 14:9-11

[2] Trevethick MA, Oakley I, Clayton NM, Strong P. Non-steroidal anti-inflammatory drug-induced gastric damage in experimental animals: underlying pathological mechanisms Gen Pharmacol. 1995 Nov;26(7):1455-9. Review

[3] .Sala A, Zarini S, Bolla M. Leukotrienes: lipid bioeffectors of inflammatory reactions Biochemistry (Mosc). 1998 Jan;63(1):8492. Review.

[4] Nyman S, Schroeder HE, Lindhe J. Suppression of inflammation and bone resorption by indomethacin during experimental periodontitis in dogs. J Periodontol. 1979 Sep;50(9):450-61

[5] Williams RC, Jeffcoat MK, Howell TH, et al. Altering the progression of human alveolar bone loss with the non-steroidal antiinflammatory drug flurbiprofen. JPeriodontol 1989;60:485-490.1

[6] Williams RC, Jeffcoat MK, Howell TH, et al. Ibuprofen: An inhibitor of alveolar bone resorption in beagles. J Periodont Res 1988;23:225-229.

[7] Howell TH, Fiorellini JP, Weber HP, Williams RC. Effect of the NSAID piroxicam, topically administered, on the development of gingivitis in beagle dogs. J Periodont Res 1991;26:180-183.

[8] Li KL, Vogel R, Jeffcoat MK, et al. The effects of ketoprofen creams on periodontal disease in rhesus monkeys. J Periodont Res $1996 ; 31: 525-532$

[9] Waite IM, Saxton CA, Young A, Wagg BJ, Corbett M. The periodontal status of subject receiving non-steroidal antiinflammatory drugs. J Periodontal Res. 1981;16(1):100-8.

[10] Williams RC, Jeffcoat MK, Howell TH, et al. Indomethacin or flurbiprofen treatment of periodontitis in beagles: Comparison of effect of bone loss. J Periodont Res 1987;22:403-407.

[11] Jeffcoat MK, Howell TH, et al. Altering the progression of human alveolar bone loss with the non-steroidal anti-inflammatory drug flurbiprofen. J Periodontol 1989;60:485-490.

[12] Howell TH, Jeffcoat MK, Goldhaber P, et al. Inhibition of alveolar bone loss in beagles with the NSAID naproxen. $J$ Periodont Res 1991;26:498-501.

[13] Yavuzyilmaz E, Yamalik N, Bulut S, Ozen S, Ersoy F, Saatçi U The gingival crevicular fluid interleukin-1 beta and tumour necrosis factor-alpha levels in patients with rapidly progressive periodontitis. Aust Dent J. 1995 Feb;40(1):46-9

[14] Stashenko P, Fujiyoshi P, Obernesser MS, Prostak L, Haffajee AD,Socransky SS. Levels of interleukin-1b in tissue from sites of active periodontal disease. J Clin Periodontol 1991; 18:548-54.

[15] Kinane DF, Winstanley FP, Adonogianaki E, Moughal NA. Bioassay of interleukin-1 (IL-1) in human gingival crevicular fluid during experimental gingivitis. Arch Oral Biol 1992; 37:153 -6.

[16] Dongari-Bagtzoglou AI, Ebersole JL. Production of inflammatory mediators and cytokines by human gingival fibroblasts following bacterial challenge. J Periodontal Res 1996;31:90-8.

[17] Hendley TM, Steed RB, Galbraith GMP. Interleukin-1b gene expression in human oral polymorphonuclear leukocytes. $J$ Periodontol 1995;66:761-5.

[18] DuFour A, Baran C, Langkamp HL, Piesco NP, Agarwal S. Regulation of differentiation of gingival fibroblasts and periodontal ligament cells by rhIL-1b and rhTNF-a. J Periodontal Res 1993;28:566-8.

[19] Ohshima M, Otsuka K, Suzuki K. Interleukin-1b stimulates collagenase production by cultured human periodontal ligament fibroblasts. J Peridodontal Res 1994;29:421-9.

[20] Okamatsu Y, Kobayashi M, Nishihara T, Hasegawa K. Interleukin-1a produced in human gingival fibroblasts induces several activities related to the progression of periodontitis by direct contact. J Periodontal Res 1996;31:355-64.

[21] Irwin CR, Schor SL, Ferguson MWJ. Effects of cytokines on gingival fibroblasts in vitro are modulated by the extracellular matrix. J Periodontal Res 1994;29:309-17.

[22] Martuscelli G, Fiorellini JP, Crohin CC, Howell TH. J Periodontol. 2000 ;71(4):573-8. The effect of interleukin-11 on the progression of ligature-induced_periodontal disease in the beagle dog. 
[23] Trepicchio WL, Bozza M, Pednault G, et al. Recombinant human interleukin-11 attenuates the inflammatory response through downregulation of proinflammatory cytokine release and nitric oxide production. J Immunol 1996;157:3627-3634.

[24] Allen JB, Wong HL, Costa GL, Bienkowski M, Wahl SM. Suppression of monocyte function and differential regulation of IL-1 and IL-1ra by IL-4 contribute to resolution of experimental arthritis. J Immunol 1993; 151:4344-4351.

[25] Martuscelli G, Fiorellini JP, Crohin CC, Howell TH. J Periodontol. 2000 ;71(4):573-8. The effect of interleukin-11 on the progression of ligature-induced periodontal disease in the beagle dog.

[26] Baer PN. Actisite (tetracycline hydrochloride periodontal fiber): a critique.

[27] Periodontal Clin Investig. 1994 Fall;16(2):5-7.

[28] Salvi GE, Lang NP. Host response modulation in the management of periodontal diseases. J Clin Periodontol 2005: 32: 108129.

[29] El-Shinnawi UM, El-Tantawy SI. The effect of alendronate sodium on alveolar bone loss in periodontitis (clinical trial). $J$ Int Acad Periodontol 2003: 5: 5-10.

[30] Shoji K, Horiuchi H, Shinoda H. Inhibitory effects of a bisphosphonate (risedronate) on experimental periodontitis in rats. $J$ Periodontal Res 1995: 30: 277-284.

[31] Weinreb M, Quartuccio H, Seedor JG, Aufdemorte TB, Brunsvold M, Chaves E, Kornman KS, Rodan GA. Histomorphometrical analysis of the effects of the bisphosphonate alendronate on bone loss caused by experimental periodontitis in monkeys. $J$ Periodontal Res 1994: 29: 35-40.

[32] Serhan CN. Lipoxins and novel aspirin-triggered 15-epilipoxins (ATL): A jungle of cell-cell interactions or a therapeutic opportunity? Prostaglandins 1997;53:107-137.

[33] Pouliot M, Serhan C.N, Lipoxin A4 and aspirin-triggered 15-epi-LXA4 inhibit tumor necrosis factor- $\alpha$-initiated neutrophil responses and trafficking: novel regulators of cytokine-chemokine axis relevant to periodontal diseases. J Periodont Res 1999; 34: 370-373.

[34] Kim D.M., Koszeghy K.L., Badovinac R.L., Kawai T., Hosokawa I, Howell, T.H. The effect of aspirin on gingival crevicular fluid levels of inflammatory and anti-inflammatory mediators in patients with gingivitis. J Periodontol 2007; 78: 1620-1626.

[35] Pouliot M, Clish CB, Petasis NA, VanDyke TE, Serhan CN. Lipoxin A4 analogues inhibit leukocyte recruitment to Porphyromonas gingivalis: A role for cyclooxygenase-2 and lipoxins in periodontal disease. Biochemistry 2000;39:4761-4768.

[36] Sodek J, Overall C. Matrix metalloproteinases in periodontal tissue remodeling. Matrix 1992;(suppl.):352-362.

[37] Kornman K.S, Page R.C, Tonetti M.S. Host response to the microbial challenge in periodontitis: assembling the players. Periodontol 2000 1997; 14:33-53.

[38] Golub LM, McNamara TF, Ryan ME, et al. Adjunctive treatment with subantimicrobial doses of doxycycline: Effects on gingival fluid collagenase activity and attachment loss in adult periodontitis. J Clin Periodontol 2001;28:146-156. 